\title{
Trajectories of Fatigue in Patients with Breast Cancer Before, During, and After Radiation Therapy
}

\author{
Anand Dhruva, MD', Marylin Dodd, RN, $\mathrm{PhD}^{2}$, Steven M. Paul, $\mathrm{PhD}^{2}$, Bruce A. Cooper, \\ $\mathrm{PhD}^{2}$, Kathryn Lee, RN, PhD $^{2}$, Claudia West, RN, $\mathbf{M S}^{2}$, Bradley E. Aouizerat, $\mathrm{PhD}^{2}$, Patrick \\ S. Swift, MD $^{3}$, William Wara, $\mathbf{M D}^{1}$, and Christine Miaskowski, RN, $\mathbf{P h D}^{2}$ \\ ${ }^{1}$ School of Medicine, University of California, San Francisco, CA \\ ${ }^{2}$ School of Nursing, University of California, San Francisco, CA \\ ${ }^{3}$ Alta Bates Comprehensive Cancer Center, Berkley, CA
}

\section{Abstract}

Background-Fatigue is a significant problem associated with radiation therapy (RT).

Objective-This study examined how evening and morning fatigue changed from the time of simulation to four months after the completion of RT and investigated whether specific demographic and disease characteristics and baseline severity of symptoms predicted initial levels of fatigue and characteristics of the trajectories of fatigue.

Methods-Seventy-three women with breast cancer completed questionnaires that assessed sleep disturbance, depression, anxiety, and pain prior to the initiation of RT and the Lee Fatigue Scale, over six months. Descriptive statistics and hierarchical linear modeling were used for data analysis.

Results-Large amounts of inter-individual variability were found in the trajectories of fatigue. Evening fatigue at baseline was negatively influenced by having children at home and depression. The trajectory of evening fatigue was worse for women who were employed. Morning fatigue at baseline was influenced by younger age, lower body mass index, and the degree of sleep disturbance and trait anxiety. Trajectories of morning fatigue were worse for patients with a higher disease stage and more medical co-morbidities.

Conclusions-Inter-individual and diurnal variability in fatigue found in women with breast cancer is similar to that found in a men with prostate cancer. However, the predictors of interindividual variability in fatigue between these two cohorts were different.

Implications for Practice-Diurnal variability and different predictors for morning and evening fatigue suggest different underlying mechanisms. The various predictors of fatigue need to be considered in the design of future intervention studies.

\section{Keywords}

fatigue; breast cancer; radiation therapy; hierarchical linear modeling; symptom patterns; symptom trajectories; sleep disturbance; depression

Address correspondence to: Christine Miaskowski, RN, PhD, FAAN, Professor and Associate Dean for Academic Affairs, Department of Physiological Nursing, University of California, 2 Koret Way - Box 0610, San Francisco, CA 94143-0610, 415-476-9407 (phone), 415-476-8899 (fax), chris.miaskowski@ nursing.ucsf.edu.

Publisher's Disclaimer: This is a PDF file of an unedited manuscript that has been accepted for publication. As a service to our customers we are providing this early version of the manuscript. The manuscript will undergo copyediting, typesetting, and review of the resulting proof before it is published in its final citable form. Please note that during the production process errors may be discovered which could affect the content, and all legal disclaimers that apply to the journal pertain. 


\section{INTRODUCTION}

Fatigue is the most common and disruptive symptom reported by patients undergoing radiation therapy (RT). $1^{-} 9$ Despite major advances in RT techniques, fatigue remains a significant clinical problem. $9^{-} 11$ In fact, fatigue associated with RT occurs in approximately $80 \%$ of patients and has deleterious effects on their functional status and quality of life (QOL). ${ }^{8}, 12^{-}$ 14

\section{Longitudinal studies of RT-induced fatigue in patients with breast cancer}

Several studies have evaluated for changes in fatigue in women with breats cancer during the course of RT. ${ }^{4,15,16}$ While the exact measurement times differed across these studies, in most cases fatigue severity ratings increased significantly from the beginning to the middle of RT and remained elevated from the middle to the end of RT. In the seven studies that evaluated for changes in fatigue in women with breast cancer following the completion of RT, ${ }^{17-23}$ fatigue severity scores decreased to pretreatment levels within 4 to 8 weeks following the completion of treatment.

\section{Predictors of RT-induced fatigue in patients with breast cancer}

Findings across seven studies that evaluated for predictors of RT-induced fatigue in women with breast cancer $16^{-} 21,23$ are inconsistent. For example, in one study, ${ }^{16}$ fatigue severity scores were not associated with stage of disease, type of breast cancer surgery, or pretreatment with chemotherapy (CTX). In contrast, Donovan and colleagues ${ }^{15}$ found that women who received CTX prior to the initiation of RT reported higher fatigue severity scores that persisted throughout RT than women who had not received CTX. In another study, 17 while fatigue severity scores were not influenced by age, stage of disease, time since surgery, weight, or length of time since diagnosis, women with higher levels of psychological and symptom distress reported higher fatigue severity scores. This finding contrasts with that of Geinitz and colleagues 23 who found no relationship between fatigue severity and anxiety and depression. In another study that examined factors that predicted fatigue severity at the end of RT,19 younger age, higher level of education, increased level of treatment related side effects and living alone were associated with higher fatigue scores. In a study of women who underwent adjuvant treatment for breast cancer, ${ }^{20}$ higher pretreatment levels of fatigue were associated with higher levels of mood disturbance, higher total network support, lower satisfaction with social support, lower morning cortisol, and higher levels of interleukin1- $\beta$. No associations were found between fatigue severity scores prior to adjuvant therapy and age, income, educational level, stage of disease, type of surgery, or type of adjuvant therapy. Finally, in a recent study of fatigue in patients with breast cancer who underwent adjuvant therapy, ${ }^{21}$ the magnitude and correlates of response shift in the measurement of fatigue were evaluated. Of note, clinically significant response shifts in ratings of fatigue did occur over time. Larger response shifts were found in women who reported higher pretreatment ratings of fatigue, in those who received CTX, and in those who reported lower fatigue catastrophizing scores.

\section{Summary and Rationale for the Proposed Study}

While the number of longitudinal studies of RT-related fatigue in women with breast cancer has increased, additional research is warranted because of the inconsistent findings across these studies. While some of these inconsistencies may be related to the measures used to assess fatigue; the timing of the measures; large variations in sample size; and/or the number and types of predictor variables tested, clinical experience as well as the findings reported to date, suggest that a large amount of inter-individual variability exists in patients' experiences of fatigue during and following RT. 
Another factor that may contribute to the inconsistent findings is the time of day that fatigue was assessed. While diurnal variations in fatigue were reported in the general population, ${ }^{24-}$ 26 only two studies have assessed for diurnal variations in fatigue in oncology patients. ${ }^{27,} 28$ In the only study of women with breast cancer, ${ }^{27}$ their ratings of fatigue were compared to those of age-matched groups of healthy women and women with benign breast disease. While breast cancer survivors reported higher levels of fatigue relative to the other two groups of women, no group differences in the diurnal pattern of fatigue was evident. Fatigue scores were lowest at 10AM and highest at 9PM. An evaluation of diurnal variations in fatigue severity might provide insights into its etiology or mechanisms. ${ }^{27-29}$

In an attempt to overcome some of the limitations noted above, as part of a larger study of symptoms in patients who underwent RT and their family caregivers (FCs), evening and morning fatigue trajectories were evaluated in patients with prostate cancer13 and in their FCs30 using a more sophisticated method for longitudinal data analysis (i.e., hierarchical linear modeling (HLM).

In this study, patients and their FCs were taught to complete the fatigue measure before going to bed each night (evening fatigue) and upon arising each morning (morning fatigue). Assessments were done prior to the initiation of RT, weekly during the course of RT, every two weeks for two months, and once a month for two months. While mean evening and morning fatigue scores were in the moderate range, a large amount of inter-individual variability was found in the trajectories of morning and evening fatigue reported by both patients and their FCs. ${ }^{13,30}$

Younger men and those with higher levels of sleep disturbance reported higher levels of evening fatigue at the initiation of RT. In terms of predictors of the trajectories of evening fatigue, younger age, lower baseline levels of evening fatigue, and higher baseline levels of sleep disturbance were associated with the worst evening fatigue trajectory. In terms of morning fatigue, the two variables that predicted higher baseline levels of morning fatigue in these patients with prostate cancer were younger age and higher baseline levels of depression and sleep disturbance. In terms of predictors of the trajectories of morning fatigue, younger age, lower baseline levels of morning fatigue, and higher baseline levels of sleep disturbance and depression were associated with the worst morning fatigue trajectory. ${ }^{13}$

In terms of the $\mathrm{FCs},{ }^{30}$ the two predictors of higher baseline levels of evening fatigue were higher baseline levels of sleep disturbance in the FC and higher baseline levels of evening fatigue in the patient. FCs with the worst evening fatigue trajectory were those who reported lower baseline levels evening fatigue, higher baseline levels of sleep disturbance, and who cared for a patient who reported higher baseline levels of evening fatigue. The three predictors of higher baseline levels of morning fatigue in these FCs were higher baseline levels of trait anxiety in the FC, lower baseline levels of family support, and higher baseline levels of morning fatigue in the patient. FCs with the worst morning fatigue trajectory were those who reported higher baseline levels of anxiety, lower baseline levels of family support and who cared for a patient who reported higher baseline levels of morning fatigue.

Findings from these two studies suggest that diurnal variation exists in patients and FCs ratings of evening and morning fatigue, that evening and morning fatigue scores in both groups were in the moderate range, and that a large amount of inter-individual variability exists in ratings of evening and morning fatigue. However, the predictors of baseline levels of evening and morning fatigue, as well as the predictors of changes in fatigue trajectories over time were different between the two measures of fatigue, as well as between patients and their FCs. ${ }^{13 \text {, }}$

${ }^{30}$ Based on these findings and because this type of longitudinal analysis, is lacking in women with breast cancer, the purposes of this study, in a sample of women who underwent RT for 
breast cancer, were: to examine how ratings of evening and morning fatigue changed from the time of simulation to four months after the completion of RT and to investigate whether specific patient, disease, and symptom characteristics predicted the initial levels of fatigue and/or characteristics of the trajectories of evening and morning fatigue.

\section{METHODS}

\section{Participants and Settings}

This analysis, drawn from the larger, descriptive, longitudinal study of symptoms in patients and their FCs, analyzed data from 73 women with breast cancer. Patients were eligible to participate if they: were adults ( $>18$ years of age); were able to read, write, and understand English; had a Karnofsky Performance Status (KPS) score of $\geq 60$ and were scheduled to receive adjuvant RT. Patients were excluded if they had metastatic disease, more than one cancer diagnosis, or had a diagnosed sleep disorder. They were recruited from RT departments located in a Comprehensive Cancer Center and a community-based oncology program. The study was approved by the Human Subjects Committee at the University of California, San Francisco and at the second study site.

One hundred and thirty-four patients with breast cancer were approached and 73 consented to participate in this longitudinal study (54.5\% response rate). The major reasons for refusal were: being too overwhelmed with their cancer experience or too busy. No differences were found in any of the demographic or disease characteristics between patients who did and did not choose to participate in this study.

\section{Study Instruments and Procedures}

The study instruments included a demographic questionnaire, the Karnofsky Performance Status (KPS) scale, 31 the Lee Fatigue Scale (LFS),32 the General Sleep Disturbance Scale (GSDS),33 the Center for Epidemiological Studies-Depression Scale (CES-D),34 the Spielberg State-Trait Anxiety Inventories (STAI-S and STAI-T),35 and a descriptive numeric rating scale (NRS) for worst pain intensity from the Brief Pain Inventory.36

The demographic questionnaire provided information on age, marital status, years of education, living arrangements, ethnicity, and employment status. In addition, patients completed a checklist of comorbidities.

Fatigue severity was measured using the 13 -item LFS. Each item is rated using a 0 to 10 NRS and a total score is calculated as the mean of the 13 items that can range from 0 to 10, with higher scores indicating higher levels of fatigue severity. Respondents were asked to rate each item based on how they felt "right now", within 30 minutes of awakening (i.e., morning fatigue) and prior to going to bed (i.e., evening fatigue) for two consecutive days and nights. The LFS has been used with healthy individuals as well as in patients with cancer and HIV.14,33,37 It was chosen for the current study because it is relatively short and easy to administer and has established cutoff scores for clinically significant levels of fatigue (i.e., $\geq 3.2$ for morning fatigue, $\geq 5.6$ for evening fatigue). The LFS has well established validity and reliability.32, 38 In the current study, the Cronbach's alphas for the LFS for evening and morning ratings were 0.95 and 0.96 , respectively.

The GSDS consists of 21 items that evaluate various aspects of sleep disturbance. Each item is rated on a NRS that ranges from 0 (never) to 7 (every day) and the 21 items are summed to yield a total score that can range from 0 (no disturbance) to 147 (extreme sleep disturbance). A cutoff score of $\geq 43.0$ indicates a clinically significant level of sleep disturbance. The GSDS has well-established validity and reliability in shift workers, pregnant women, and patients 
with cancer and HIV. ${ }^{14,33,39,40}$ In the current study, the Cronbach's alpha for the GSDS total score was 0.81 .

The CES-D consists of 20 items selected to represent the major symptoms in the clinical syndrome of depression. Scores can range from 0 to 60 , with scores of $\geq 16$ indicating the need for individuals to seek clinical evaluation for major depression. The CES-D has well established concurrent and construct validity. ${ }^{34,41,42}$ In the current study, the Cronbach's alpha for the CES-D was 0.83 .

The STAI-T and STAI-S inventories consist of 20 items each that are rated from 1 to 4 . The scores for each scale are summed and can range from 20 to 80 . A higher score indicates greater anxiety. The cutoff scores for clinically significant levels of trait and state anxiety are $\geq 31.8$ and $\geq 32.2$, respectively. The STAI-T measures an individual's predisposition to anxiety determined by his/her personality and estimates how a person generally feels. The STAI-S measures an individual's transitory emotional response to a stressful situation. It evaluates the emotional responses of worry, nervousness, tension, and feelings of apprehension related to how a person feels "right now" in a stressful situation. The STAI-S and STAI-T inventories have well established criterion and construct validity and internal consistency reliability coefficients. ${ }^{35,43,44}$ In the current study, the Cronbach's alphas for the STAI-T and STAI-S were 0.86 and 0.91 , respectively.

Worst pain was evaluated using a descriptive NRS that ranged from 0 (no pain) to 10 (excruciating pain). A descriptive numeric rating scale is a valid and reliable measure of pain intensity. ${ }^{45}$ Because the majority of the patients in this study did not have pain, for the subsequent longitudinal analyses, pain was recoded as present or absent.

\section{Study Procedures}

At the time of the simulation visit (i.e., approximately one week prior to the start of RT), patients were approached by a research nurse to discuss participation in the study. After obtaining written informed consent, they were asked to complete the baseline study questionnaires. Patients were taught to complete the LFS prior to going to bed each night (i.e., evening fatigue) and upon arising each morning (i.e., morning fatigue) for two consecutive days. Assessments were done at the time of the simulation visit (baseline), weekly during the course of RT, every 2 weeks for 2 months, and once a month for 2 months following RT. The majority of the patients completed 16 assessments.

\section{Data Analysis}

Descriptive statistics and frequency distributions were generated on the sample characteristics and baseline symptom severity scores using SPSS ${ }^{\mathrm{TM}}$ Version 15.0. For each of the 16 fatigue assessments, a mean score for each of the LFS scores (i.e., two consecutive assessments of evening and morning fatigue) was calculated for use in the subsequent statistical analyses.

HLM, based on full maximum likelihood estimation, was done using the software developed by Raudenbush and colleagues. ${ }^{46}$ The repeated measures of fatigue were conceptualized as being nested within individuals. Compared with other methods of analyzing change, HLM has two major advantages. First, HLM can accommodate unbalanced designs which allows for the analysis of data when the number and the spacing of the assessments vary across respondents. Although every patient was to be assessed on a pre-specified schedule, the actual number of assessments was not the same for all of the patients because some patients had longer periods of RT and some had scheduling conflicts. Second, HLM has the ability to model individual change, which helps to identify more complex patterns of change that are often overlooked by other methods. ${ }^{46,47}$ 
With HLM, the repeated measures of the outcome variables (i.e., evening and morning fatigue) are nested within individuals and the analysis of change in fatigue scores has two levels: within persons (Level 1) and between persons (Level 2). At Level 1, the outcome is conceptualized as varying within individuals and is a function of person-specific change parameters plus error. At Level 2, these person-specific change parameters are multivariate outcomes that vary across individuals. These Level 2 outcomes can be modeled as a function of demographic or clinical characteristics that vary between individuals, plus an error associated with the individual. Combining Level 1 with Level 2 results in a mixed model with fixed and random effects. ${ }^{46}$, 48,49

Separate HLM analyses were done to evaluate changes over time in ratings of evening and morning fatigue. Each HLM analysis proceeded in two stages. First, intra-individual variability in fatigue over time was examined. In this study, time in weeks, refers to the length of time from the simulation visit to four months after the completion of RT (i.e., six months with a total of 16 assessments). Three Level 1 models, which represented that the patients' fatigue levels (a) did not change over time (i.e., no time effect), (b) changed at a constant rate (i.e., linear time effect), and (c) changed at a rate that accelerates or decelerates over time (i.e., quadratic effect) were compared. At this point, the Level 2 model was constrained to be unconditional (i.e., no predictors) and likelihood ratio tests were used to determine the best model. These analyses answered the first research question and identified the change parameters that best described individual changes in evening and morning fatigue over time.

The second stage of the HLM analysis, which answered the second research question, examined inter-individual differences in the trajectories of evening and morning fatigue by modeling the individual change parameters (i.e., intercept, linear and quadratic slopes) as a function of proposed predictors at Level 2 . Table 1 presents a list of the proposed predictors that was developed based on a review of the literature of fatigue in women with breast cancer who underwent RT. ${ }^{15-18,20-23}$ To improve estimation efficiency and construct a model that was parsimonious, an exploratory Level 2 analysis was done in which each potential predictor was assessed to see if it would result in a better fitting model if it alone was added as a Level 2 predictor. Predictors with a t-value of $<2.0$, which indicates a lack of a significant effect, were dropped from subsequent model testing. All of the potentially significant predictors from the exploratory analyses were entered into the model to predict each individual change parameter. Only predictors that in conjunction with other variables were retained in the final model. A pvalue of $<.05$ indicates statistical significance.

\section{RESULTS}

\section{Patient Characteristics and Symptom Severity Scores}

The demographic, disease, and treatment characteristics of the 73 patients are presented in Table 2. This sample of patients, with a mean age of 55 years, was well-educated, had a KPS score of 87.7 , an average of 5 co-morbidities, and $22 \%$ were caring for children at home. The most common co-morbid conditions were allergies (58.6\%), back problems (54.8\%), headaches (44.4\%), and hypertension (27.8\%). Fifty-six percent had localized disease (stage 1) while $44 \%$ had locally advanced (stage 2 or 3 ) disease. Seventy-four percent of the patients had breast conserving surgery. Almost 50\% had a lymph node dissection and 55\% had received CTX prior to RT. The mean baseline symptom severity scores for the 73 patients are listed in Table 2.

\section{Individual and Mean Change in Evening and Morning Fatigue}

The first HLM analyses examined how evening and morning levels of fatigue changed from the time of the simulation visit to 4 months after the completion of RT. Two models were 
estimated in which the function of time was linear and quadratic. For both evening fatigue and morning fatigue, the goodness-of-fit tests of the deviance between the linear and quadratic models indicated that a quadratic model fit the data significantly better than a linear model (both $\mathrm{p}<0.0001$ ).

Evening Fatigue-The estimates of the quadratic change model are presented in Table 3 (unconditional model). Because the model had no covariates (i.e., unconditional), the intercept represents the estimated amount of evening fatigue (i.e., 4.95 on a 0 to 10 scale) at the time of the simulation visit. The estimated linear rate of change in evening fatigue, for each additional week, was $0.072(\mathrm{p}=0.002)$ and the estimated quadratic rate of change per week was -0.002 $(\mathrm{p}=0.009)$. The weighted combination of the linear and quadratic terms defines each curve. Figure 1 displays the trajectory for evening fatigue from the time of the simulation visit to 4 months after the completion of RT. Evening fatigue increased over the course of RT (i.e., weeks 0 to 9 ) and then declined after the completion of RT. It should be noted that the mean fatigue scores for the various groups depicted in all of the figures are estimated or predicted means based on the HLM analyses.

Morning Fatigue-As shown in Table 3, in the unconditional model, the intercept represents the estimated amount of morning fatigue (i.e., 2.9) at the time of the simulation visit. The estimated linear rate of change in morning fatigue, for each additional week, was 0.049 $(p=0.055)$ and the estimated quadratic rate of change per week was $-0.003(p<0.01)$. Figure 1 displays the trajectory for morning fatigue from the time of the simulation visit to 4 months after the completion of RT. Morning fatigue decreased over the course of RT and then plateaued after the completion of RT.

Although the results indicate a sample-wide increase followed by a decrease in evening fatigue and a gradual decrease in morning fatigue followed by a plateau, they do not imply that all patients exhibited the same trajectories. The variance in individual change parameters estimated by the models (i.e., variance components, Table 3) suggested that substantial interindividual differences existed in the trajectories of evening and morning fatigue which are illustrated in Figure 2. These results suggested that further examination of inter-individual differences in the individual change parameters was warranted.

\section{Inter-individual Differences in the Trajectories of Evening and Morning Fatigue}

The second stage of the HLM analyses tested the hypothesis that the pattern of change over time in evening and morning fatigue varied based on specific person, disease, treatment, and/ or symptom variables that were found to influence fatigue levels of patients who underwent RT for breast cancer. Exploratory analyses were done with the potential predictors listed in Table 1. To improve estimation efficiency and construct models that were parsimonious, exploratory Level 2 analyses were done in which each potential predictor was assessed to see if it would result in a better fitting model if it alone was added as a Level 2 predictor. Predictors with a t-value of $<2.0$, indicating lack of a significant effect, were dropped from subsequent model testing. All of the significant predictors from the exploratory analyses were entered into the models to predict each individual change parameter. Only predictors that maintained a significant contribution in conjunction with other variables were retained in the final models.

Evening Fatigue-As shown in the final model in Table 3, the two variables that predicted inter-individual differences in the intercept for evening fatigue were having children at home and baseline level of depressive symptoms. The variable that predicted inter-individual differences in the slope parameters for evening fatigue was being employed. 
To illustrate the effects of the three different predictors on patients' trajectories of evening fatigue, Figure 3 displays the adjusted change curves of evening fatigue that were estimated based on differences in having children at home (i.e., children at home, yes or no), baseline level of depressive symptoms (i.e., low CES-D/high CES-D calculated based on 1 standard deviation (SD) above and below the mean CES-D score), and work (i.e., working or not working).

Morning Fatigue-As shown in the final model in Table 3, the four variables that predicted inter-individual differences in the intercept for morning fatigue were age, BMI, trait anxiety, and sleep disturbance. The two variables that predicted inter-individual differences in the slope parameters for morning fatigue were number of co-morbidities and stage of disease.

To illustrate the effects of the different predictors on patients' trajectories of morning fatigue, Figure 4 displays the adjusted change curves for morning fatigue that were estimated based on differences in age (i.e., younger/older calculated based on $1 \mathrm{SD}$ above and below the mean age of the patients), BMI (i.e., low/high BMI calculated based on 1 SD above and below the mean BMI score), trait anxiety (i.e. low/high trait anxiety calculated based on $1 \mathrm{SD}$ above and below the mean STAI-T score), and baseline level of sleep disturbance (i.e. low sleep/high sleep disturbance calculated based on 1 SD above and below the mean GSDS score). Figure 5 displays the adjusted change curves for morning fatigue based on predictors of the slope parameters (i.e., number of comorbidities, stage of disease).

\section{DISCUSSION}

The results of this study of women with breast cancer extend findings on both inter-individual and diurnal variability in fatigue initially reported in a group of prostate cancer patients who underwent RT.13 Diurnal variability was evaluated in both studies to test the hypothesis that fatigue severity varies over the course of the day and may be influenced by different factors. Mean levels of morning (2.9) and evening (5.0) fatigue at the initiation of RT were in the moderate range.50,51 However, these levels of fatigue may be underestimates of the amount of fatigue women who are about to undergo RT experience because among the 61 patients who declined to participate in this study, the primary reason cited was being too overwhelmed with the experience of cancer. In addition, these baseline levels of morning and evening fatigue were higher than those reported by men with prostate cancer at the initiation of RT (i.e., 2.0 and 3.6, respectively). 13

The incremental increases in evening and morning fatigue in this sample as a whole were modest but consistent with previous reports. ${ }^{4,} 15,16,18,23$ However, the use of HLM, compared to more traditional statistical approaches (e.g., repeated measures analysis of variance) provided evidence of a large amount of inter-individual variability in baseline levels, as well as in the trajectories of evening and morning fatigue. In addition, these HLM analyses provide insights into which of these women with breast cancer were at increased risk for more severe and prolonged fatigue trajectories.

The estimated mean evening fatigue scores at the time of the simulation visit ranged from 0.2 to 8.2 , which spans the mild to severe range. ${ }^{50,51}$ Consistent with previous reports that evaluated overall levels of fatigue during RT, $4,15,16,18,23$ mean evening fatigue scores increased gradually during RT and gradually declined following the completion of RT (Figure 1). The predictors of baseline levels of evening fatigue included having children at home and higher CES-D scores. In addition, women who were employed showed a small but steady increase in evening fatigue over the six months of the study. As shown in Figures 2A and 2B, patients who were caring for children at home and those who reported higher levels of depressive symptoms had evening fatigue scores at the initiation of RT that were above the 
clinically significant level of 5.6. ${ }^{32,51}$ While previous reports have not examined the impact of caring for children on women's level of fatigue during RT, the finding of increased fatigue in women who reported higher depression scores at the initiation of RT is consistent with two previous reports. ${ }^{17,20}$ However, the finding that being employed was a predictor of higher levels of evening fatigue is not consistent with a previous report that found that women who worked more hours reported lower fatigue severity scores. ${ }^{22}$ This inconsistency warrants consideration in future studies.

Taking care of children and working may represent important lifestyle factors that contribute to the development of fatigue in women who are receiving RT for breast cancer. While these factors were not identified as predictors of evening fatigue in men with prostate cancer, ${ }^{13}$ clinicians who care for women with breast cancer should assess for these factors and counsel patients about possible lifestyle modifications or the need for assistance while on treatment.

Estimated mean morning fatigue scores at the time of the simulation visit ranged from 0.0 to 7.5. In contrast to evening fatigue, morning fatigue scores decreased slightly during RT and then plateaued about two weeks after the completion of RT (Figure 1).The predictors of higher baseline levels of morning fatigue were younger age, higher levels of sleep disturbance and trait anxiety, and lower BMI. Both younger age and higher levels of sleep disturbance were predictors of baseline levels of morning fatigue in men with prostate cancer who underwent RT. ${ }^{13}$ Of note, other cross-sectional studies of oncology patients have associated sleep disturbance ${ }^{52-54}$ and trait anxiety ${ }^{55}$ with higher levels of average fatigue.

At the time of the simulation visit, the mean sleep disturbance and anxiety scores were above the cutoffs for clinically significant levels of these two symptoms. ${ }^{35,51,56}$ In addition, as shown in Figures 3C and 3D, patients with higher levels of trait anxiety or sleep disturbance had morning fatigue scores at the initiation of RT that were above the clinically significant level of 3.2. ${ }^{32,51}$ These findings suggest that clinicians need to assess not only for fatigue but for anxiety and sleep disturbance in patients with breast cancer who undergo RT.

In terms of BMI being a predictor of baseline levels of morning fatigue, $4.1 \%$ of the women in this study were underweight and $37.8 \%$ had a BMI in the normal range. This finding warrants additional investigation because the data on the relationship between BMI and fatigue are inconclusive. ${ }^{18,57,58}$

The predictors of the trajectories of morning fatigue were co-morbidity and stage of disease. A higher number of co-morbidities was associated with higher levels of fatigue, which confirms findings from one study. ${ }^{52}$ While pretreatment with CTX was associated with higher levels of fatigue in some studies, ${ }^{15,21}$ but not in others, ${ }^{16}$ it was not a predictor of morning or evening fatigue in this study. However, in this study, advanced stage of disease may be a proxy for a higher level of treatment burden and/or an independent disease-related predictor of higher levels of fatigue. All of these predictors warrant investigation in future studies.

Diurnal variability in and different predictors for evening and morning fatigue suggest that different factors and different mechanisms underlie the development of fatigue. While morning fatigue appears to be more affected by biologic factors, evening fatigue appears to be more affected by behavioral factors. These data suggest that different interventions may be needed to reduce diurnal variations in fatigue.

Different predictors for fatigue were found for this cohort of women with breast cancer compared to a cohort of patients with prostate cancer who underwent RT. ${ }^{13}$ The patients with prostate cancer were more homogeneous in their clinical characteristics. All of these patients had localized prostate cancer, a low Gleason score, and a similar treatment history. The patients with breast cancer were more heterogeneous in terms of their disease stage and treatment 
history. These between group differences may explain why a number of biologic factors were related to fatigue in the breast cancer cohort but not in the prostate cancer cohort. While these findings warrant replication, they suggest that interventions for fatigue may need to be individualized based on the patient's cancer diagnosis, as well as on personal and clinical characteristics.

Some limitations of this study include the relatively small sample size and the fact that most of the study participants were white and highly educated, which might limit the generalizability of the findings. The strengths of this study include the collection of longitudinal data on the trajectories of fatigue and the use of HLM to identify predictors of fatigue. The identification of factors that influence the development of fatigue may assist clinicians to identify patients at highest risk for the development of this deleterious symptom. In addition, these factors may be used in the development of intervention studies for cancer-related fatigue. However, additional research is warranted, with larger samples, to fully characterize those phenotypic and genotypic characteristics that influence patients' experiences of fatigue during RT.

\section{Acknowledgments}

This research was supported by a grant from the National Institute of Nursing Research (NR04835). Dr. Aouizerat is funded through the National Institutes of Health Roadmap for Medical Research Grant (K12RR023262). Additional support for the corresponding author's program of research was provided through unrestricted grants from Endo Pharmaceuticals, PriCara, Unit of Ortho-McNeil, Inc. and Purdue Pharma LP. The assistance of the research nurses on the project, Carol Maroten and Mary Cullen, and the support from the physicians and nurses at the study sites were greatly appreciated.

\section{REFERENCES}

1. Hickok JT, Roscoe JA, Morrow GR, Mustian K, Okunieff P, Bole CW. Frequency, severity, clinical course, and correlates of fatigue in 372 patients during 5 weeks of radiotherapy for cancer. Cancer 2005;104(8):1772-1778. [PubMed: 16116608]

2. Jereczek-Fossa BA, Marsiglia HR, Orecchia R. Radiotherapy-related fatigue. Crit Rev Oncol Hematol 2002;41(3):317-325. [PubMed: 11880207]

3. Lawrence DP, Kupelnick B, Miller K, Devine D, Lau J. Evidence report on the occurrence, assessment, and treatment of fatigue in cancer patients. J Natl Cancer Inst Monogr 2004;32:40-50. [PubMed: 15263040]

4. Greenberg DB, Sawicka J, Eisenthal S, Ross D. Fatigue syndrome due to localized radiation. J Pain Symptom Manage 1992;7(1):38-45. [PubMed: 1538180]

5. Haylock PJ, Hart LK. Fatigue in patients receiving localized radiation. Cancer Nurs 1979;2(6):461467. [PubMed: 259440]

6. Irvine D, Vincent L, Graydon JE, Bubela N, Thompson L. The prevalence and correlates of fatigue in patients receiving treatment with chemotherapy and radiotherapy. A comparison with the fatigue experienced by healthy individuals. Cancer Nurs 1994;17(5):367-378. [PubMed: 7954384]

7. King KB, Nail LM, Kreamer K, Strohl RA, Johnson JE. Patients' descriptions of the experience of receiving radiation therapy. Oncol Nurs Forum 1985;12(4):55-61. [PubMed: 3847996]

8. Smets EM, Visser MR, Willems-Groot AF, Garssen B, Schuster-Uitterhoeve AL, de Haes JC. Fatigue and radiotherapy: (B) experience in patients 9 months following treatment. Br J Cancer 1998;78(7): 907-912. [PubMed: 9764582]

9. Danjoux C, Gardner S, Fitch M. Prospective evaluation of fatigue during a course of curative radiotherapy for localised prostate cancer. Support Care Cancer 2007;15(10):1169-1176. [PubMed: 17333296]

10. Truong PT, Berthelet E, Lee JC, et al. Prospective evaluation of the prevalence and severity of fatigue in patients with prostate cancer undergoing radical external beam radiotherapy and neoadjuvant hormone therapy. Can J Urol 2006;13(3):3139-3146. [PubMed: 16813705] 
11. Jereczek-Fossa BA, Santoro L, Alterio D, et al. Fatigue during head-and-neck radiotherapy: prospective study on 117 consecutive patients. Int J Radiat Oncol Biol Phys 2007;68(2):403-415. [PubMed: 17398020]

12. Curt GA. Impact of fatigue on quality of life in oncology patients. Semin Hematol 2000;37:14-17. [PubMed: 11068951]

13. Miaskowski C, Paul SM, Cooper BA, et al. Trajectories of fatigue in men with prostate cancer before, during, and after radiation therapy. J Pain Symptom Manage 2008;35(6):632-643. [PubMed: 18358683]

14. Miaskowski C, Lee KA. Pain, fatigue, and sleep disturbances in oncology outpatients receiving radiation therapy for bone metastasis: a pilot study. J Pain Symptom Manage 1999;17(5):320-332. [PubMed: 10355211]

15. Donovan KA, Jacobsen PB, Andrykowski MA, et al. Course of fatigue in women receiving chemotherapy and/or radiotherapy for early stage breast cancer. J Pain Symptom Manage 2004;28 (4):373-380. [PubMed: 15471655]

16. Lavdaniti M, Patiraki E, Dafni U, Katapodi M, Papathanasoglou E, Sotiropoulou A. Prospective assessment of fatigue and health status in Greek patients with breast cancer undergoing adjuvant radiotherapy. Oncol Nurs Forum 2006;33(3):603-610. [PubMed: 16676016]

17. Irvine DM, Vincent L, Graydon JE, Bubela N. Fatigue in women with breast cancer receiving radiation therapy. Cancer Nurs 1998;21(2):127-135. [PubMed: 9556939]

18. Wratten C, Kilmurray J, Nash S, et al. Fatigue during breast radiotherapy and its relationship to biological factors. Int J Radiat Oncol Biol Phys 2004;59(1):160-167. [PubMed: 15093912]

19. Knobf MT, Sun Y. A longitudinal study of symptoms and self-care activities in women treated with primary radiotherapy for breast cancer. Cancer Nurs 2005;28(3):210-218. [PubMed: 15915066]

20. Von Ah DM, Kang DH, Carpenter JS. Predictors of cancer-related fatigue in women with breast cancer before, during, and after adjuvant therapy. Cancer Nurs 2008;31(2):134-144. [PubMed: 18490890]

21. Andrykowski MA, Donovan KA, Jacobsen PB. Magnitude and correlates of response shift in fatigue ratings in women undergoing adjuvant therapy for breast cancer. J Pain Symptom Manage 2009;37 (3):341-351. [PubMed: 18757176]

22. Poirier P. The relationship of sick leave benefits, employment patterns, and individual characteristics to radiation therapy-related fatigue. Oncol Nurs Forum 2006;33(3):593-601. [PubMed: 16676015]

23. Geinitz H, Zimmermann FB, Stoll P, et al. Fatigue, serum cytokine levels, and blood cell counts during radiotherapy of patients with breast cancer. Int J Radiat Oncol Biol Phys 2001;51(3):691698. [PubMed: 11597810]

24. Cahill CA. Differential diagnosis of fatigue in women. J Obstet Gynecol Neonatal Nurs 1999;28(1): 81-86.

25. Sonnenschein M, Sorbi MJ, van Doornen LJ, Schaufeli WB, Maas CJ. Electronic diary evidence on energy erosion in clinical burnout. J Occup Health Psychol 2007;12(4):402-413. [PubMed: 17953498]

26. Lee KA. Sleep and fatigue. Annu Rev Nurs Res 2001;19:249-273. [PubMed: 11439783]

27. Curran SL, Beacham AO, Andrykowski MA. Ecological momentary assessment of fatigue following breast cancer treatment. J Behav Med 2004;27(5):425-444. [PubMed: 15675633]

28. Dimsdale JE, Ancoli-Israel S, Ayalon L, Elsmore TF, Gruen W. Taking fatigue seriously, II: variability in fatigue levels in cancer patients. Psychosomatics 2007;48(3):247-252. [PubMed: 17478594]

29. Hacker ED, Ferrans CE. Ecological momentary assessment of fatigue in patients receiving intensive cancer therapy. J Pain Symptom Manage 2007;33(3):267-275. [PubMed: 17349496]

30. Fletcher BA, Schumacher KL, Dodd M, et al. Trajectories of fatigue in family caregivers of patients undergoing radiation therapy for prostate cancer. Res Nurs Health 2009;32:125-139. [PubMed: 19072846]

31. Karnofsky, D. Performance Scale. New York: Plenum Press; 1977.

32. Lee KA, Hicks G, Nino-Murcia G. Validity and reliability of a scale to assess fatigue. Psychiatry Res 1991;36(3):291-298. [PubMed: 2062970] 
33. Lee KA, DeJoseph JF. Sleep disturbances, vitality, and fatigue among a select group of employed childbearing women. Birth 1992;19(4):208-213. [PubMed: 1472269]

34. Radloff LS. The CES-D Scale: A self-report depression scale for research in the general population. The CES-D Scale: A self-report depression scale for research in the general population. Applied Psychol Measure 1977;1(3):385-401.

35. Spielberger, CG.; Gorsuch, RL.; Suchene, R.; Vagg, PR.; Jacobs, GA. Manual for the State-Anxiety (Form Y): Self Evaluation Questionnaire. Palo Alto, CA: Consulting Psychologists Press; 1983.

36. Daut RL, Cleeland CS, Flanery RC. Development of the Wisconsin Brief Pain Questionnaire to assess pain in cancer and other diseases. Pain 1983;17(2):197-210. [PubMed: 6646795]

37. Lee KA, Portillo CJ, Miramontes $\mathrm{H}$. The fatigue experience for women with human immunodeficiency virus. J Obstet Gynecol Neonatal Nurs 1999;28(2):193-200.

38. Lee KA, Lentz MJ, Taylor DL, Mitchell ES, Woods NF. Fatigue as a response to environmental demands in women's lives. Image J Nurs Sch 1994;26(2):149-154. [PubMed: 8063323]

39. Lee KA. Self-reported sleep disturbances in employed women. Sleep 1992;15(6):493-498. [PubMed: 1475563]

40. Lee KA, Portillo CJ, Miramontes H. The influence of sleep and activity patterns on fatigue in women with HIV/AIDS. J Assoc Nurses AIDS Care 2001;(12 Suppl):19-27. [PubMed: 11563234]

41. Sheehan TJ, Fifield J, Reisine S, Tennen H. The measurement structure of the Center for Epidemiologic Studies Depression Scale. J Pers Assess 1995;64(3):507-521. [PubMed: 7760258]

42. Carpenter JS, Andrykowski MA, Wilson J, et al. Psychometrics for two short forms of the Center for Epidemiologic Studies-Depression Scale. Issues Ment Health Nurs 1998;19(5):481-494. [PubMed: 9782864]

43. Kennedy BL, Schwab JJ, Morris RL, Beldia G. Assessment of state and trait anxiety in subjects with anxiety and depressive disorders. Psychiatr Q 2001;72(3):263-276. [PubMed: 11467160]

44. Bieling PJ, Antony MM, Swinson RP. The State-Trait Anxiety Inventory, Trait version: structure and content re-examined. Behav Res Ther 1998;36(7-8):777-788. [PubMed: 9682533]

45. Jensen MP. The validity and reliability of pain measures in adults with cancer. J Pain 2003;4(1):221. [PubMed: 14622723]

46. Raudenbush, S.; Bryk, A. Hierarchical Linear Models: Applications and Data Analysis Methods. 2 ed. Thousand Oaks, CA: Sage Publications; 2002.

47. Raudenbush SW. Comparing personal trajectories and drawing causal inferences from longitudinal data. Annu Rev Psychol 2001;52:501-525. [PubMed: 11148315]

48. Li LW. Longitudinal changes in the amount of informal care among publicly paid home care recipients. Gerontologist 2005;45(4):465-473. [PubMed: 16051909]

49. Li LW. From caregiving to bereavement: trajectories of depressive symptoms among wife and daughter caregivers. J Gerontol B Psychol Sci Soc Sci 2005;60(4):P190-P198. [PubMed: 15980286]

50. Mendoza TR, Wang XS, Cleeland CS, et al. The rapid assessment of fatigue severity in cancer patients: use of the Brief Fatigue Inventory. Cancer 1999;85(5):1186-1196. [PubMed: 10091805]

51. Fletcher BS, Paul SM, Dodd MJ, et al. Prevalence, severity, and impact of symptoms on female family caregivers of patients at the initiation of radiation therapy for prostate cancer. J Clin Oncol 2008;26 (4):599-605. [PubMed: 18235118]

52. Bower JE, Ganz PA, Desmond KA, Rowland JH, Meyerowitz BE, Belin TR. Fatigue in breast cancer survivors: occurrence, correlates, and impact on quality of life. J Clin Oncol 2000;18(4):743-753. [PubMed: 10673515]

53. Yennurajalingam S, Palmer JL, Zhang T, Poulter V, Bruera E. Association between fatigue and other cancer-related symptoms in patients with advanced cancer. Support Care Cancer 2008;27:27.

54. Servaes P, Verhagen S, Bleijenberg G. Determinants of chronic fatigue in disease-free breast cancer patients: a cross-sectional study. Ann Oncol 2002;13(4):589-598. [PubMed: 12056710]

55. De Vries J, Van der Steeg AF, Roukema JA. Trait anxiety determines depressive symptoms and fatigue in women with an abnormality in the breast. Br J Health Psychol 2008;7:7.

56. Lee KA, Gay CL. Sleep in late pregnancy predicts length of labor and type of delivery. Am J Obstet Gynecol 2004;191(6):2041-2046. [PubMed: 15592289] 
57. Andrykowski MA, Curran SL, Lightner R. Off-treatment fatigue in breast cancer survivors: a controlled comparison. J Behav Med 1998;21(1):1-18. [PubMed: 9547419]

58. Yang YH. Relationship between fatigue and nutritional status in patients with cancer undergoing radiotherapy. Taehan Kanho Hakhoe Chi 2003;33(4):478-487. [PubMed: 15314423] 


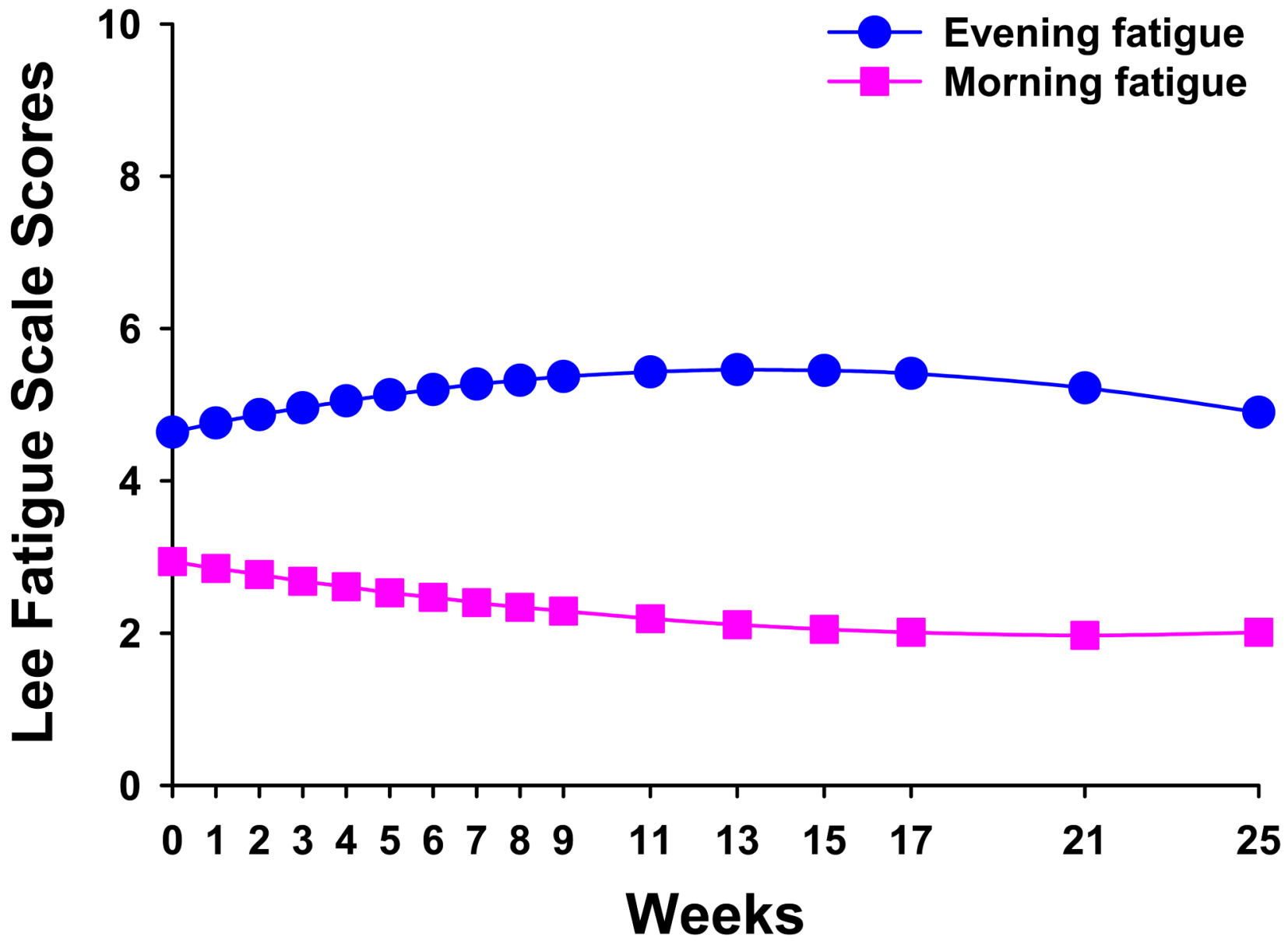

Figure 1.

Trajectories of evening and morning fatigue over the 25 weeks of the study 
A

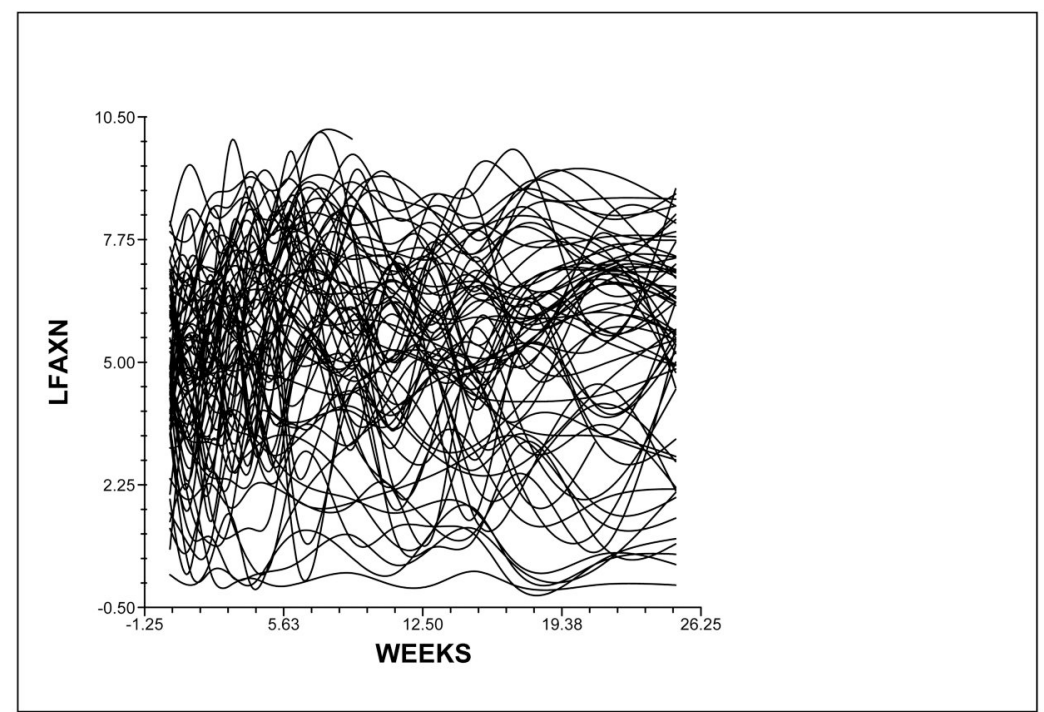

B

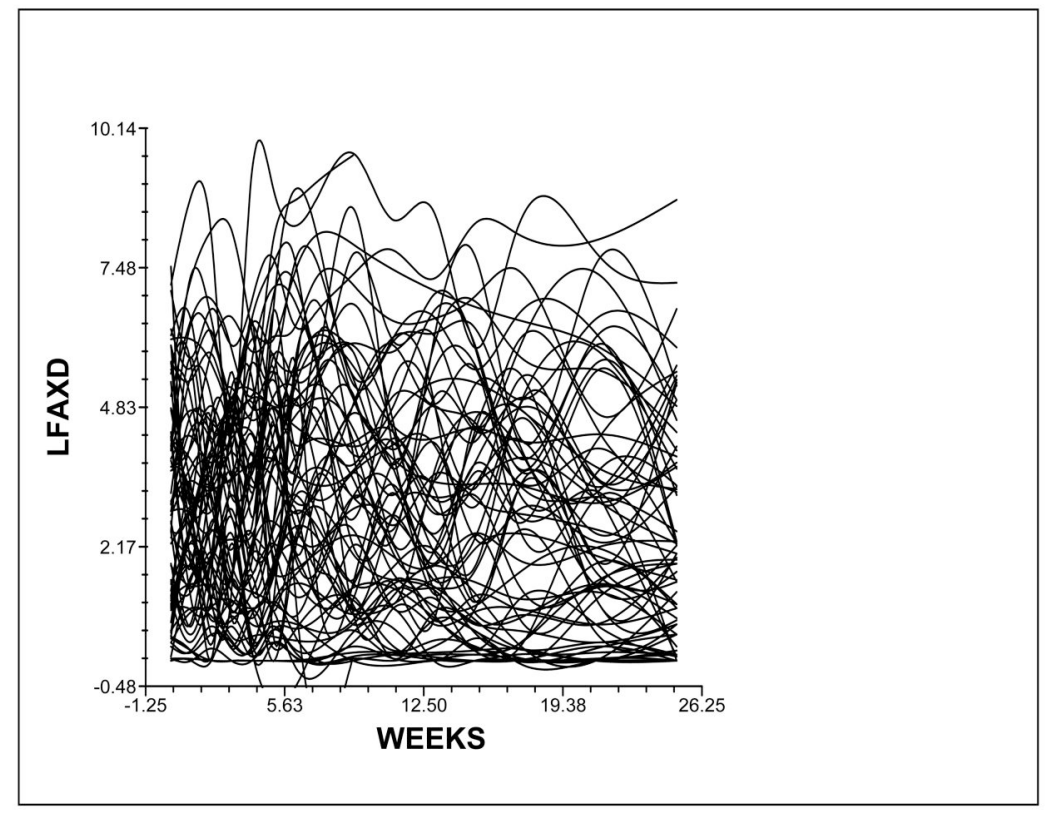

Figure 2.

Spaghetti plots of the 73 patients' individual evening (A) and morning (B) fatigue trajectories over the 25 weeks of the study. 
A.

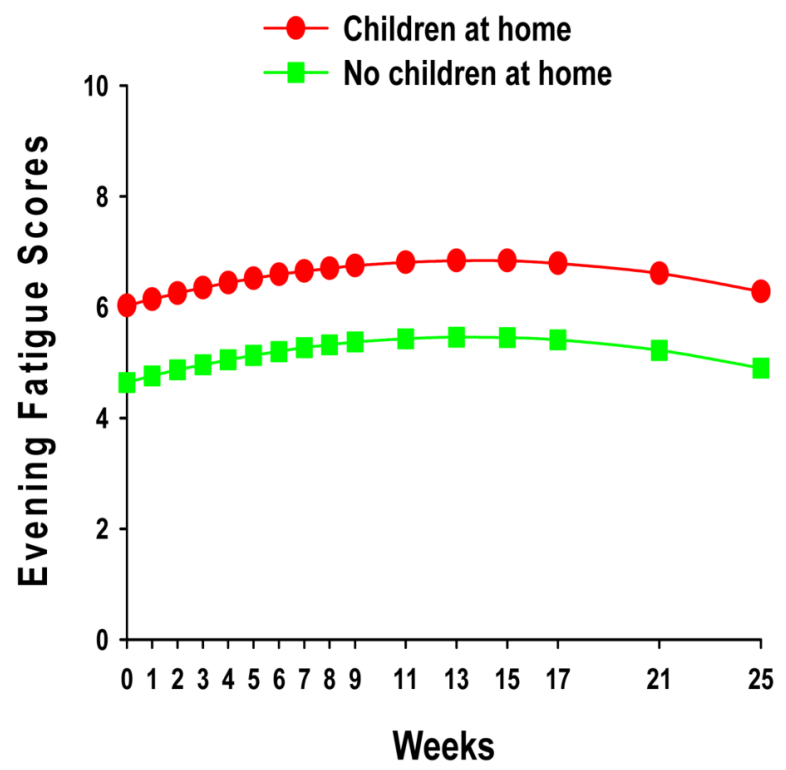

B.

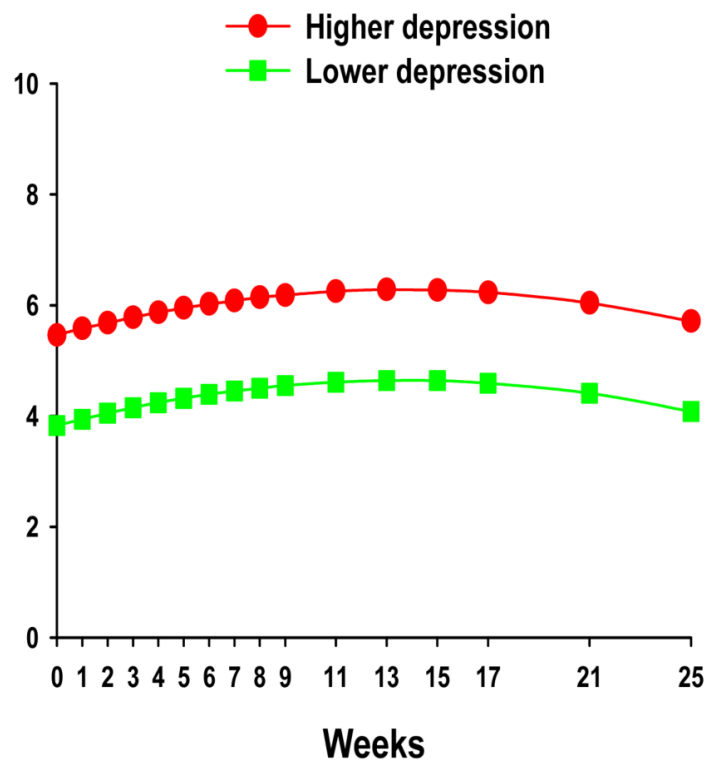

C.

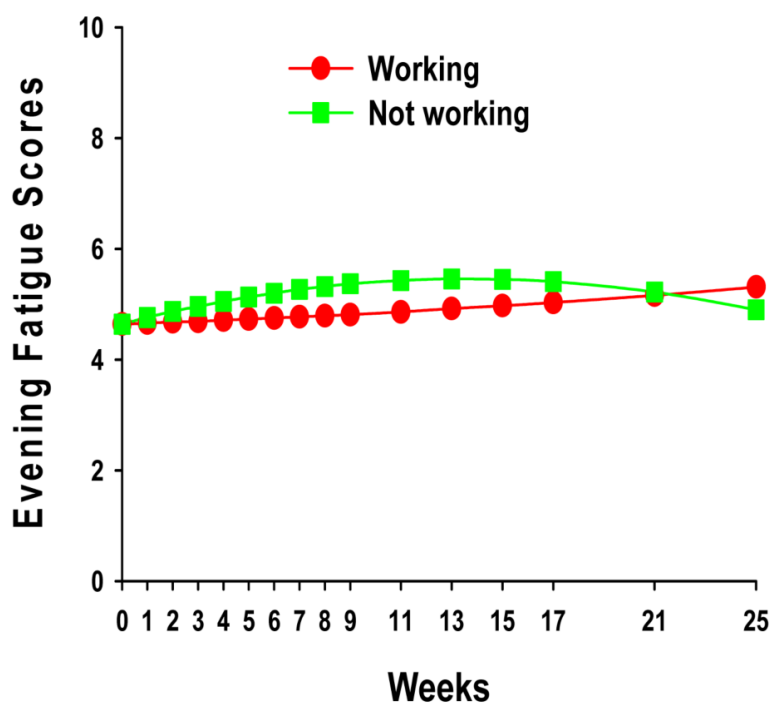

Figure 3.

Influence of having children at home (Figure 3A) and baseline levels of depression (Figure 3B) on inter-individual differences in the intercept for evening fatigue and influence of being employed (Figure 3C) on the slope parameters for evening fatigue. 
A.

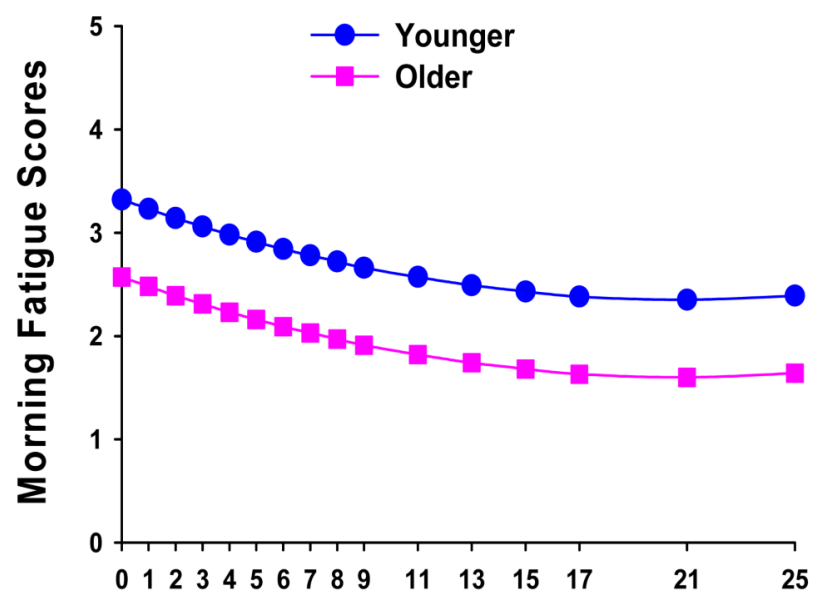

B.

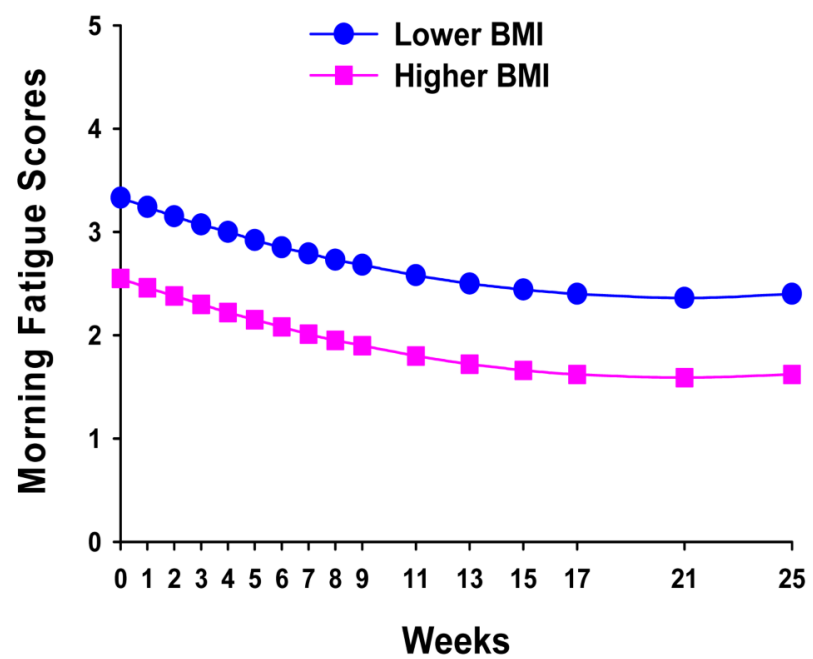

C.

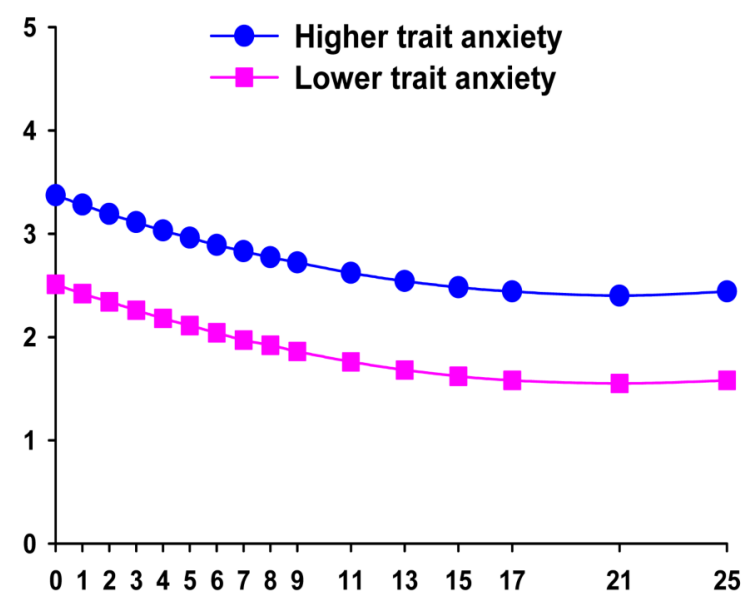

D.

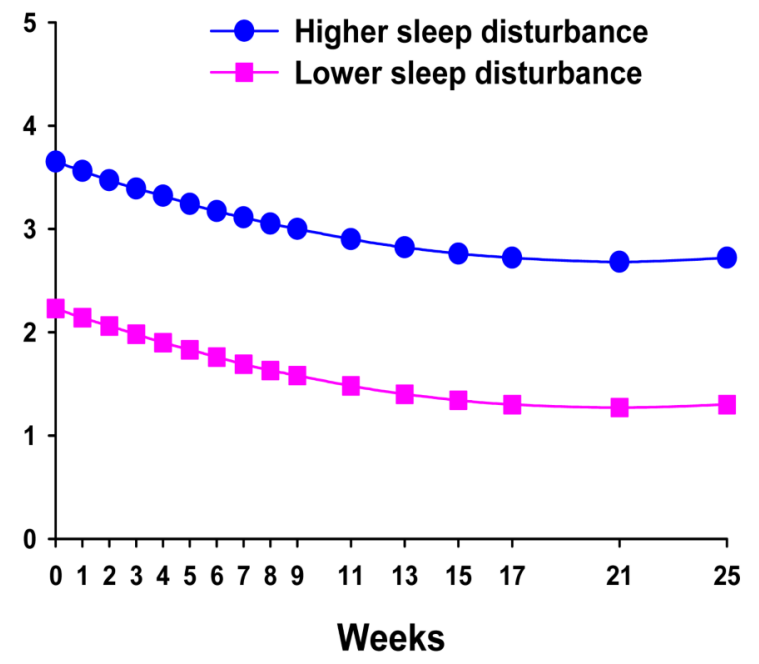

Figure 4.

Influence of age (Figure 4A), body mass index (Figure 4B), trait anxiety score (Figure 4C), and sleep disturbance score (Figure 4D) on inter-individual differences in the intercept for morning fatigue. 
A.
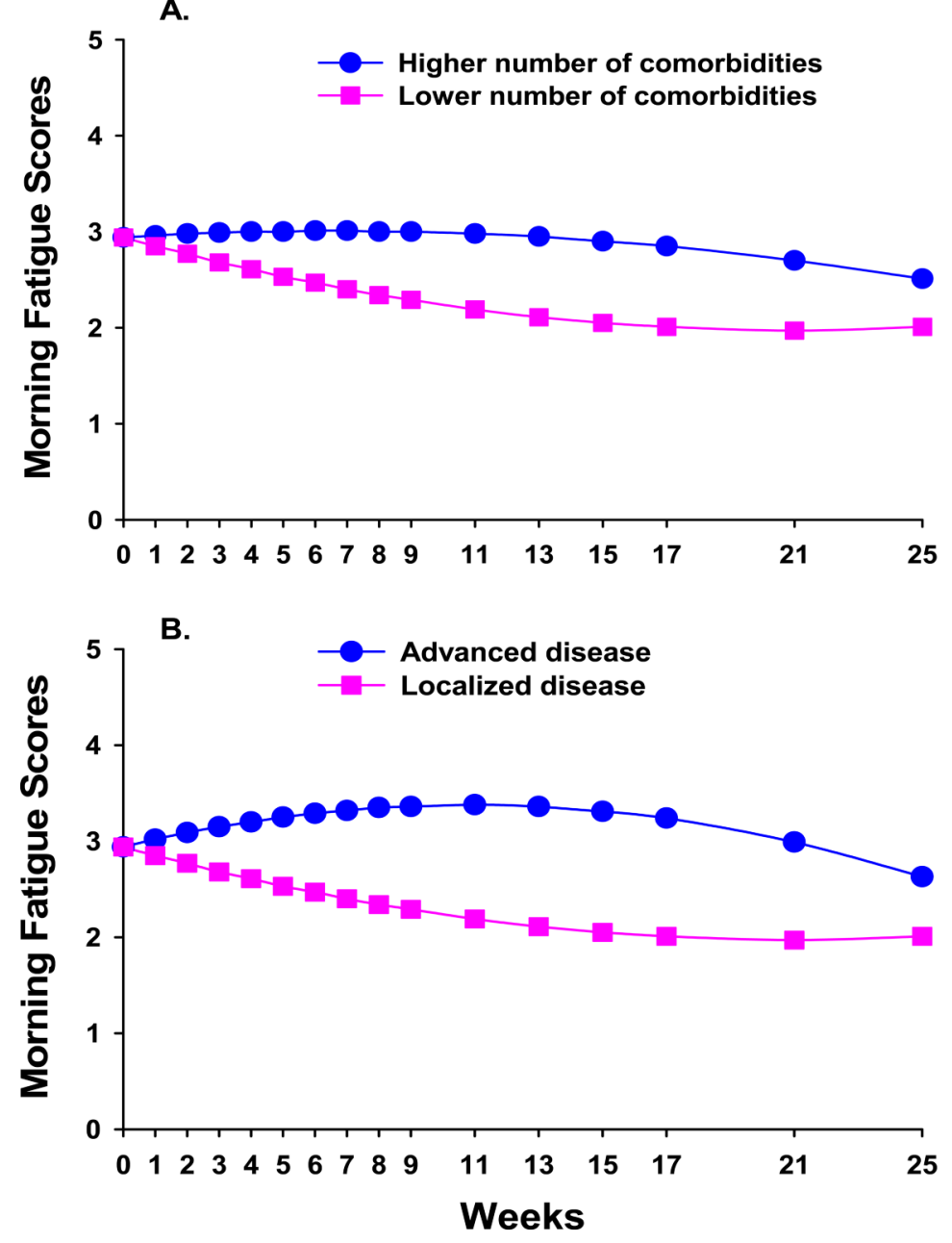

Figure 5.

Influence of number of comorbidities (Figure 5A) and stage of disease (Figure 5B) on the slope parameters for morning fatigue. 
0
00
0
00
0
0
0
0
0
0
0
0
0
0
0
0
0
0
0
0
0
0
0
0
0
0
0
0
0
0
0
0
0
0
0
0

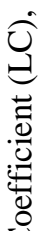

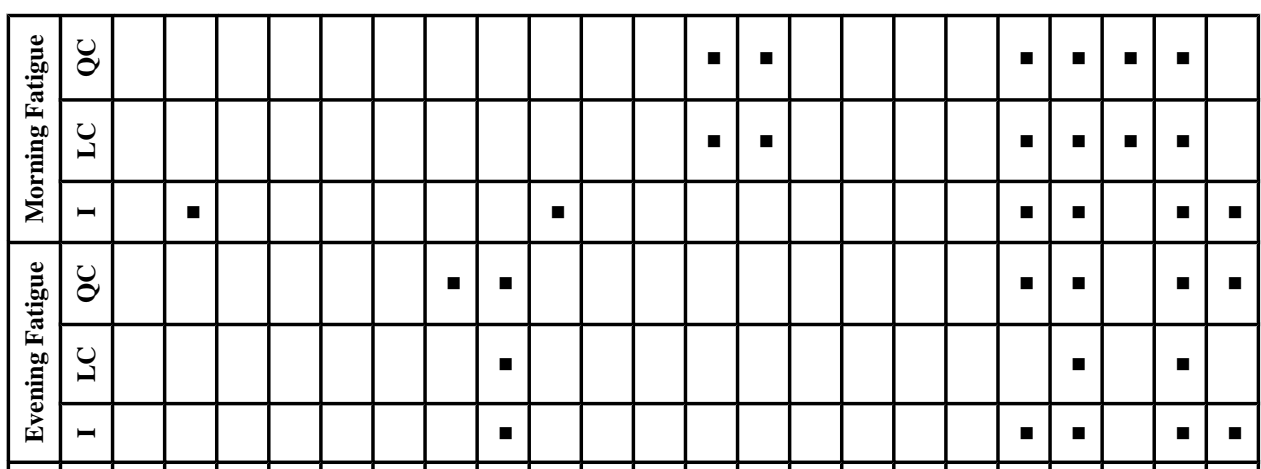


Table 2

Demographic, Disease, and Treatment Characteristics of the Patients $(n=73)$

\begin{tabular}{|c|c|}
\hline Characteristic & Mean (Standard Deviation) \\
\hline Age (years) & $55.1(11.0)$ \\
\hline Education (years) & $16.2(2.7)$ \\
\hline Karnofsky Performance Status Score & $87.7(12.4)$ \\
\hline Number of comorbidities & $5.3(2.6)$ \\
\hline Weight (pounds) & $165.2(43.6)$ \\
\hline Body mass index & $27.4(7.3)$ \\
\hline Hemoglobin (g/dl) & $12.7(1.2)$ \\
\hline Hematocrit $(\%)$ & $37.5(3.4)$ \\
\hline Lives alone & $41.0 \%$ \\
\hline \multicolumn{2}{|l|}{ Marital status } \\
\hline Married/partnered & $28.8 \%$ \\
\hline Divorced/separated & $30.1 \%$ \\
\hline Other & $41.1 \%$ \\
\hline \multicolumn{2}{|l|}{ Ethnicity } \\
\hline Non-white & $30.0 \%$ \\
\hline White & $70.0 \%$ \\
\hline \multicolumn{2}{|l|}{ Employed } \\
\hline Yes & $45.0 \%$ \\
\hline No & $55.0 \%$ \\
\hline Children at home & $22.0 \%$ \\
\hline \multicolumn{2}{|l|}{ Stage } \\
\hline Localized & $56.2 \%$ \\
\hline Locally advanced & $43.8 \%$ \\
\hline Any chemotherapy received & $55.0 \%$ \\
\hline Lymph node dissection & $49.0 \%$ \\
\hline Hormonal therapy prior to diagnosis & $44.0 \%$ \\
\hline Total dose of RT (cGys) & $5829(438.3)$ \\
\hline \multicolumn{2}{|c|}{ Mean symptom severity scores at baseline } \\
\hline LFS score for evening fatigue & $4.9(1.8)$ \\
\hline LFS score for morning fatigue & $2.9(2.1)$ \\
\hline
\end{tabular}




\begin{tabular}{|l|c|}
\hline Characteristic & Mean (Standard Deviation) \\
\hline GSDS score & $44.7(21.7)$ \\
CES-D score & $12.0(9.2)$ \\
Trait Anxiety Inventory score & $36.2(11.3)$ \\
State Anxiety Inventory score & $33.7(12.9)$ \\
\hline Percentage of patients with pain & $49.3 \%$ \\
\hline
\end{tabular}

Abbreviations: CES-D = Center for Epidemiologic Studies Depression Scale, GSDS = General Sleep Disturbance Scale, LFS = Lee Fatigue Scale, $\mathrm{RT}=$ radiation therapy 
Table 3

Hierarchical Linear Models of Evening and Morning Fatigue

\begin{tabular}{|c|c|c|c|}
\hline \multicolumn{2}{|l|}{ Evening Fatigue } & \multicolumn{2}{|c|}{ Coefficient (SE) } \\
\hline \multicolumn{2}{|l|}{ Variable } & $\begin{array}{c}\text { Unconditional } \\
\text { Model }\end{array}$ & Final Model \\
\hline \multicolumn{4}{|l|}{ Fixed Effects } \\
\hline \multicolumn{2}{|l|}{ Intercept } & $4.947(0.214)^{* *}$ & $4.644(0.203)^{* *}$ \\
\hline \multicolumn{2}{|c|}{$\operatorname{Time}^{a}$ (linear rate of change) } & $0.072(0.218)^{*}$ & $0.120(0.028)^{* *}$ \\
\hline \multicolumn{2}{|c|}{ Time $^{2}$ (quadratic rate of change) } & $-0.002(0.0008)^{*}$ & $-0.004(0.001)^{* *}$ \\
\hline \multicolumn{4}{|c|}{ Time invariant covariates } \\
\hline \multirow[t]{2}{*}{ Intercept: } & Children at home & & $1.384(0.427)^{*}$ \\
\hline & CES-D score & & $0.089(0.019)^{* *}$ \\
\hline Linear: & Work $\times$ time & & $-0.105(0.042)^{+}$ \\
\hline Quadratic: & Work $\times$ time $^{2}$ & & $-0.005(0.002)^{*}$ \\
\hline \multicolumn{4}{|c|}{ Variance components } \\
\hline \multicolumn{2}{|l|}{ In intercept } & $2.979^{* *}$ & $1.989^{* *}$ \\
\hline \multicolumn{2}{|c|}{ In linear rate } & $0.015^{* *}$ & $0.012^{* *}$ \\
\hline \multicolumn{2}{|c|}{ In quadratic fit } & $0.00001^{*}$ & $0.00001^{+}$ \\
\hline \multirow{2}{*}{\multicolumn{2}{|c|}{$\begin{array}{l}\text { Goodness-of-fit deviance(parameters estimated) } \\
\text { Model comparison }\left(\chi^{2}[d f]\right)\end{array}$}} & $3154.432(10)$ & $3117.1853(14)$ \\
\hline & & & $37.247(4)^{* *}$ \\
\hline \multicolumn{2}{|l|}{ Morning Fatigue } & \multicolumn{2}{|c|}{ Coefficient (SE) } \\
\hline \multicolumn{2}{|l|}{ Variable } & $\begin{array}{l}\text { Unconditional } \\
\text { Model }\end{array}$ & Final Model \\
\hline \multicolumn{4}{|l|}{ Fixed Effects } \\
\hline \multicolumn{2}{|l|}{ Intercept } & $2.940(0.212)^{* *}$ & $2.942(0.155)^{* *}$ \\
\hline \multicolumn{2}{|c|}{ Time $^{a}$ (linear rate of change) } & $0.049(0.025)^{+}$ & $-0.093(0.038)^{+}$ \\
\hline \multicolumn{2}{|c|}{ Time $^{2}$ (quadratic rate of change) } & $-0.003(0.001)^{*}$ & $-0.002(0.001)$ \\
\hline \multicolumn{4}{|c|}{ Time invariant covariates } \\
\hline \multirow[t]{4}{*}{ Intercept: } & Age & & $-0.034(0.014)^{+}$ \\
\hline & Body Mass Index & & $-0.054(0.021)^{*}$ \\
\hline & Trait anxiety score & & $0.038(0.016)^{+}$ \\
\hline & GSDS score & & $0.033(0.008)^{* *}$ \\
\hline \multirow[t]{2}{*}{ Linear: } & Number of co-morbidities $\times$ time & & $0.112(0.042)^{+}$ \\
\hline & Stage of disease $\times$ time & & $0.173(0.0424)^{* *}$ \\
\hline Quadratic: & Number of co-morbidities $\times$ time $^{2}$ & & $-0.004(0.002)^{+}$ \\
\hline
\end{tabular}




\begin{tabular}{|c|c|c|}
\hline \multirow{2}{*}{$\begin{array}{l}\text { Evening Fatigue } \\
\text { Variable }\end{array}$} & \multicolumn{2}{|c|}{ Coefficient (SE) } \\
\hline & $\begin{array}{c}\text { Unconditional } \\
\text { Model }\end{array}$ & Final Model \\
\hline Stage of disease $\times$ time $^{2}$ & & $-0.006(0.002)^{*}$ \\
\hline \multicolumn{3}{|l|}{ Variance components } \\
\hline In intercept & $2.900^{*}$ & $1.389^{* *}$ \\
\hline In linear rate & $0.027^{*}$ & $0.014^{* *}$ \\
\hline In quadratic fit & $0.00003^{*}$ & $0.00001^{*}$ \\
\hline Goodness-of-fit deviance(parameters estimated) & $3131.8235(10)$ & $3057.1598(18)$ \\
\hline Model comparison $\left(\chi^{2}[d f]\right)$ & & $74.664(8)^{* *}$ \\
\hline
\end{tabular}

${ }^{a}$ Time was coded 0 at the time of the simulation visit

**

$\mathrm{p}<0.0001$

$*$

$\mathrm{p}<0.01$

${ }^{+} \mathrm{p} \leq 0.05$

Abbreviations: CES-D = Center for Epidemiologic Studies Depression Scale, GSDS = General Sleep Disturbance Scale, LFS = Lee Fatigue Scale, $\mathrm{RT}=$ radiation therapy 